later (w16), with respect to the 5 clustering PROs as well as for coping behavior, illness perceptions and social support.

Results: Analyses revealed 3 distinct clusters. Details are depicted in Table 1. Of 140 patients, $77.9 \%$ were assigned to the 'concordant to disease activity' cluster, $9.3 \%$ to the 'dominant fatigue' cluster and $12.9 \%$ to the 'dominant pain and fatigue' cluster. When comparing the concordant cluster with both discordant clusters, differences at BL and w16 were shown, with small effects on patients' evaluation of their disease at year 1 , such as more negative beliefs about the consequences of RA at w16 in patients reporting dominant pain and fatigue at year 1. Few differences with medium effects were identified, such as a moderately higher use of avoidance as a coping style at $\mathrm{w} 16$ and a stronger belief that symptoms can be explained by RA at w16, in patients reporting dominant fatigue at year 1 compared to patients in the concordant cluster. Large differences between patients in concordant and discordant clusters were found for pain and fatigue levels, which were already higher after 4 months of treatment for those patients reporting persistent pain and fatigue at year

Abstract THU0103 -Table 1.

Table 1. Mean scores at year 1 of the patient-reported outcomes used to assign patients to a cluster ( $n=140$ persistent responders)

\begin{tabular}{lccc}
\hline \multicolumn{4}{c}{ Clusters } \\
\hline & $\begin{array}{c}\text { Concordant to } \\
\text { disease activity } \\
\mathrm{n}=109(77.9 \%)\end{array}$ & $\begin{array}{c}\text { Dominant fatigue } \\
\text { Pain (VAS 0-100) }\end{array}$ & $\begin{array}{c}\text { Dominant pain and } \\
\text { fatigue } \\
\mathrm{n}=18(9.3 \%)\end{array}$ \\
Fatigue (VAS 0-100) & 7 & 15 & 45 \\
Functionality (HAQ 0-3) & 0.07 & 70 & 42 \\
RA-related quality of life & 3.92 & 0.24 & 0.26 \\
(RAQoL 0-30) & & 6.21 & 5.55 \\
Sleep quality (PSQl 0-21) & 5 & 6 & 5 \\
\hline Note. RA: Rheumatoid arthritis. VAS: Visual Analog Scale; HAQ: Health Assessment Questionnaire;
\end{tabular}

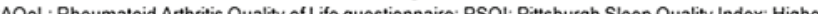
scores indicate worse pain, worse fatigue, more functional loss, worse health-related quality of life and worse sleep quality. Clinical response was defined as achieving a disease activity score (DAS28CRP) $<2.6$. Persistent response was defined as an early clinical response at week 16 , sustained between week 16 and year 1 .

Conclusion: Three distinct groups of persistent responders with early RA were identified based on their 1-year PRO profile. The majority of patients were well-synchronized, reporting very low pain and fatigue levels in concordance with their well-controlled disease activity. One in 5 persistent responders, however, seemed to have unmet needs. In view of early identification of patients at risk of poor wellbeing despite good disease control, early pain and fatigue levels and certain coping behavior and illness perceptions were recognized as potential targets for future interventions.

Disclosure of Interests: Kristien Van der Elst: None declared, Patrick Verschueren Grant/research support from: Unrestricted Pfizer Grant for Early RA research, Diederik De Cock: None declared, An De Groef: None declared, Veerle Stouten: None declared, Sofia Pazmino: None declared, Johanna Vriezekolk: None declared, Johan Joly: None declared, Philip Moons: None declared, Rene Westhovens Grant/research support from: Bristol-Myers Squibb, Consultant for: Celltrion, Galapagos-Gilead

DOI: 10.1136/ANNRHEUMDIS-2019-EULAR.3629:

\section{THU0104 HIGH-PERFORMANCE CHEMICAL ISOTOPE LABELING LIQUID CHROMATOGRAPHY MASS SPECTROMETRY FOR DISCOVERY OF METABOLITE BIOMARKERS OF RHEUMATOID ARTHRITIS}

Xiaohang Wang ${ }^{1}$, Joel Paschke ${ }^{2}$, Rana Dadashova ${ }^{2}$, Edna Hutchings ${ }^{2}$, LI Liang ${ }^{1}$, Walter P Maksymowych ${ }^{2} .{ }^{1}$ University of Alberta, Chemistry, Edmonton, Canada; ${ }^{2} \mathrm{CaRE}$ Arthritis, Edmonton, Canada

Background: Early diagnosis of rheumatoid arthritis (RA) is hampered by suboptimal accuracy of currently available serological biomarkers. Recent advancements in metabolomic profiling include liquid chromatography mass spectrometry (LC-MS) and in-depth profiling of amine/phenol and carboxyl submetabolomes, resulting in 1000-fold increase in detection sensitivity, and universal metabolome-standard methodology to facilitate metabolome comparisons among different data sets.
Objectives: We aimed to identify a metabolite signature with consistently high accuracy for RA.

Methods: Sera from 2 RA cohorts were analyzed: Cohort A samples were from 50 RA patients, 39 female (mean age 49.9), 11 male (mean age 47.8), symptom duration $<3$ years, DAS $>3.7$, naïve to b-DMARD, and 50 age and sex-matched healthy controls. Cohort B samples were from 50 RA patients, 40 female (mean age 53.4), 10 male (mean age 57.2 ), symptom duration $<5$ years, samples from both pre- and post- (3 months) treatment with $\mathrm{TNFi}$ and a second set of 50 age and sexmatched healthy controls. Amine/phenol- and carboxyl-containing metabolites were labeled by ${ }^{12} \mathrm{C}$-dansyl (Dns) chloride and ${ }^{12} \mathrm{C}$-dimethylaminophenacyl (DmPA) bromide, respectively. A pooled sample was generated and labeled by ${ }^{13} \mathrm{C}$-Dns and ${ }^{13} \mathrm{C}$-DmPA, respectively. LC-UV quantification was applied for sample normalization. After mixing individual ${ }^{12} \mathrm{C}$-labeled serum with the ${ }^{13} \mathrm{C}$-labeled pool in equal amounts, the mixtures were analyzed by LC-QTOF-MS for relative quantification.

Results: A total of 3415 amine/phenol and 2114 carboxyl metabolites were commonly detected in more than $80 \%$ of the samples. For amine/ phenol submetabolome profiling, partial least squares discriminant analysis (PLSDA) showed a clear separation of the groups for each cohor ( $R 2=0.98 ; Q 2=0.92$ for cohort $A$ and $R 2=0.93 ; Q 2=0.79$ for cohort B). Simi larly, for carboxyl submetabolome profiling, a clear separation between $\mathrm{RA}$ and controls was demonstrable $(\mathrm{R} 2=0.93 ; \mathrm{Q} 2=0.80$ for cohort $\mathrm{A}$ and $\mathrm{R} 2=0.84$;Q2 $=0.55$ for cohort B). 13 positively identified amine/phenol-containing metabolites, including o-phosphoethanolamine and glycyl-valine, were both significant in cohort $A$ and cohort $B$, and each of these metabolites showed similar fold changes between RA and controls for both cohorts. 5 carboxyl-containing metabolites, with 1 positively identified azelaic acid, and 4 unidentified, were both significant in 2 cohorts. The ROC AUC $(95 \% \mathrm{Cl})$ of the 13 amine/phenol-containing metabolite panel were $0.99(0.94-1.00), 0.98(0.92-1.00)$ and $0.98(0.95-1.00)$ for cohort A and cohort B (pre- and post-treatment), respectively, with sensitivity/specificity of $94 \% / 96 \%, 94 \% / 95 \%, 94 \% / 94 \%$. The ROC AUC $(95 \% \mathrm{Cl})$ of the 5 carboxyl metabolite panel were $0.92(0.86-0.97), 0.96 \quad(0.91-0.99), 0.89$ $(0.82-0.95)$, respectively, with sensitivity/specificity of $86 \% / 86 \%, 90 \% / 91 \%$, $80 \% / 80 \%$. The combined panel of 18 metabolites demonstrated ROC AUC $>0.99$ with sensitivity and specificity $>95 \%$ for each cohort. None of these biomarker metabolites correlated with age, gender, or symptom duration.

Conclusion: Consistent discrimination in metabolite profiles between discovery and verification cohorts generated high priority candidates for further biomarker validation in RA.

Disclosure of Interests: Xiaohang Wang: None declared, Joel Paschke: None declared, Rana Dadashova: None declared, Edna Hutchings: None declared, Liang Li: None declared, Walter P Maksymowych Grant/research support from: AbbVie, Pfizer, Janssen, Novartis, Consultant for: AbbVie Eli Lilly, Boehringer, Galapagos, Janssen, Novartis, Pfizer and UCB Pharma; Chief Medical Officer for Canadian Research and Education Arthritis

DOI: 10.1136/annrheumdis-2019-eular.4474

\section{THU0105 DIVERSE DISEASE ACTIVITY MEASURES DEMONSTRATE THAT THE INITIATION OF TOFACITINIB LEADS TO SUSTAINED CLINICAL RESPONSES IN THE MAJORITY OF RHEUMATOID ARTHRITIS PATIENTS IN A CLINIC UTILIZING A TREAT TO TARGET STRATEGY}

Craig Wiesenhutter ${ }^{1,2} .{ }^{1}$ University of Washington, Department of Medicine, Seattle, United States of America; ${ }^{2}$ Coeur d'Alene Arthritis Clinic, Coeur d"Alene, United States of America

Background: Treating Rheumatoid Arthritis (RA) patients to target (T2T) has been shown to result in better outcomes in patients with RA [1] and offers the opportunity to analyze clinical responses and changes in certain laboratory parameters after treatment with specific therapeutic agents. Objectives: To determine the frequency, magnitude, and duration of clinical responses and changes in certain laboratory parameters after initiating Tofacitinib in a single community rheumatology clinic (author's) utilizing a T2T strategy.

Methods: Patients at a community based rheumatology clinic undergo disease activity measure assessments (DAMs) on a routine basis as part of the implementation of the T2T strategy. These assessments include the disease activity score in 28 joints (DAS28CRP), and the more objective 